\title{
Fixed Points of Automorphisms Permuting the Generators Cyclically in Free solvable Lie algebras
}

\author{
Zerrin Esmerligil \\ Department of Mathematics, Çukurova University, Adana, Turkey
}

\begin{abstract}
We investigate fixed points of an automorphism of a free solvable Lie algebra which permutes the generators cyclically. Let $\Theta$ be a cyclic permutation of order $n$ which belongs to the nth symmetric group $S_{n}$. We give form of the fixed points of an automorphism of a free solvable Lie algebra which is induced by the permutation $\Theta$.
\end{abstract}

Keywords: Free Solvable Lie algebra, automorphism, fixed point, cyclic permutation.

\section{I.INTRODUCTION}

Let $\mathrm{F}$ be the free Lie algebra freely generated by a set $\mathrm{X}=\left\{\mathrm{x}_{1}, \ldots, \mathrm{x}_{\mathrm{n}}\right\}, \mathrm{n} \geq 2$, over a field $\mathrm{K}$. The derived series of $\mathrm{F}$ is defined as the following:

$\delta^{0}(\mathrm{~F})=\mathrm{F}, \delta^{1}(\mathrm{~F})=\mathrm{F}^{\prime}=[\mathrm{F}, \mathrm{F}]$ and for $\mathrm{m}>1$ we define $\delta^{\mathrm{m}}(\mathrm{F})=\left[\delta^{\mathrm{m}-1}(\mathrm{~F}), \delta^{\mathrm{m}-1}(\mathrm{~F})\right]$.

Fixed points subalgebras of free Lie algebras are studied by Bryant[1] and Drensky [3]. In [2] Bryant and Papistas have obtained some results about fixed point subalgebras of relatively free Lie algebras. Later, Ekici and Sönmez [4] have given a criterion detecting nontrivial fixed points of IA- automorphisms of a free metabelian Lie algebra.

Fixed point subalgebras of automorphisms preserving the length of words of free solvable groups are described by Tomaszewski [5]. In this work we obtained corresponding results for free solvable Lie algebras.

By $\mathrm{L}_{\mathrm{m}}$ we denote the free solvable Lie algebra $\mathrm{F} / \delta^{\mathrm{m}}(\mathrm{F})$ of rank $\mathrm{n}$ and solvability class $\mathrm{m}$.

Let $\theta$ be an automorphism of $\mathrm{F}$ of order $\mathrm{k}$ induced by a permutation $\sigma \in S_{n}$, where $S_{n}$ is the nth symmetric group. The automorphism $\theta$ induces an automorphism $\bar{\theta}: \mathrm{L}_{\mathrm{m}} \rightarrow$ $\mathrm{L}_{\mathrm{m}}$ which is defined as $\bar{\theta}(\bar{\omega})=\theta(\omega)+\delta^{\mathrm{m}}(\mathrm{F})$, where $\omega \in \mathrm{F}, \quad \bar{\omega}=\omega+\delta^{\mathrm{m}}(\mathrm{F})$. For an element $\bar{\omega}$ of $\mathrm{L}_{\mathrm{m}}$ if $\bar{\theta}(\bar{\omega})=\bar{\omega}$ then $\bar{\omega}$ is called a fixed point of $\mathrm{L}$.

It can be easily seen that if $\theta$ has order $k$ then every element of the form

$$
\bar{\omega}+\bar{\theta}(\bar{\omega})+\bar{\theta}^{2}(\bar{\omega})+\ldots+\bar{\theta}^{\mathrm{k}-1}
$$

is a fixed point for $\bar{\theta}$, where $\mathrm{k} \geq 2, \bar{\omega} \in \mathrm{F} / \delta^{\mathrm{m}}(\mathrm{F})$.

It is not obvious that only such elements are the fixed points. In this work we prove that every fixed point of $\bar{\theta}$ has the form (1).

\section{MAIN RESULT}

Assume that $\sigma \in \mathrm{S}_{\mathrm{n}}$ is a product of disjoint cycles $\sigma_{\mathrm{i}}$ of length $r_{i}, i=1, \ldots$, s. Let $G=F / F^{\prime}$.

Lemma

Assume that $\theta$ is an automorphism of $F$ which is induced by $\sigma$. If $\hat{\theta}$ is an automorphism of G, induced by $\theta$ then every fixed points of $\hat{\theta}$ has the form

$$
\sum_{\mathrm{i}=1}^{\mathrm{s}}\left(\widehat{\omega}_{\mathrm{i}}+\hat{\theta}\left(\widehat{\omega}_{\mathrm{i}}\right)+\hat{\theta}^{2}\left(\widehat{\omega}_{\mathrm{i}}\right)+\cdots+\widehat{\theta}^{\mathrm{r}_{\mathrm{i}}-1}\left(\widehat{\omega}_{\mathrm{i}}\right)\right),
$$

where $\widehat{\omega}_{i}=\beta_{i} \hat{x}_{i_{1}}, \beta_{i} \in K, i=1, \ldots, s$.

Proof

Let $\hat{\theta}$ be an automorphism of G, induced by $\theta$. If $\hat{v} \in G$ is a fixed point of $\hat{\theta}$ then $\hat{\theta}(\hat{v})=\hat{v}$. The element $\hat{v}$ can be uniquely written as

$$
\hat{v}=\sum_{j=1}^{n} c_{j} \hat{x}_{j}, \quad c_{j} \in K
$$

By taking into account the cycles of $\sigma$ we arrange the generators which we see in $\hat{v}$ as $\hat{v}=\sum_{i=1}^{s} \sum_{t=1}^{r_{i}} c_{i_{t}} \hat{x}_{i_{t}}$. Using the equality $\hat{\theta}(\hat{v})=\hat{v}$ we get

$$
c_{i_{t}}=c_{i_{1}}=\beta_{i}, \quad 1 \leq t, l \leq r_{i}, \quad i=1, \ldots, s .
$$

Therefore $\hat{v}=\sum_{i=1}^{s} \beta_{i}\left(\sum_{t=1}^{r_{i}} \hat{x}_{i_{t}}\right)$. Since $\hat{\theta}\left(\hat{x}_{i_{1}}\right)=$

$$
\mathrm{x}_{\mathrm{i}_{2}}, \ldots, \hat{\theta}\left(\hat{\mathrm{x}}_{\mathrm{i}_{\mathrm{r}_{i}}}\right)=\hat{\mathrm{x}}_{\mathrm{i}_{1}} \text { then }
$$

$$
\sum_{\mathrm{t}=1}^{\mathrm{r}_{\mathrm{i}}} \hat{\mathrm{x}}_{\mathrm{i}_{\mathrm{t}}}=\left(\mathrm{I}+\hat{\theta}+\hat{\theta}^{2}+\cdots+\hat{\theta}^{\mathrm{r}_{\mathrm{i}}-1}\right)\left(\hat{\mathrm{x}}_{\mathrm{i}_{1}}\right)
$$

and so $\hat{\mathrm{v}}=\sum_{\mathrm{i}=1}^{\mathrm{s}}\left(\mathrm{I}+\hat{\theta}+\hat{\theta}^{2}+\cdots+\hat{\theta}^{\mathrm{r}_{\mathrm{i}}-1}\right)\left(\beta_{\mathrm{i}} \hat{\mathrm{x}}_{\mathrm{i}_{1}}\right)$.

By the above Lemma it is clear that if $\sigma$ is a cycle of order $\mathrm{n}$ and $\hat{\theta}$ is an automorphism of G induced by $\theta$ then every fixed point of $\hat{\theta}$ has the form

$$
\begin{gathered}
\widehat{\omega}+\hat{\theta}(\widehat{\omega})+\widehat{\theta}^{2}(\widehat{\omega})+\cdots+\widehat{\theta}^{\mathrm{n}-1}(\widehat{\omega}), \\
\text { where } \widehat{\omega}=\beta \widehat{\mathrm{x}}_{\mathrm{k}}, \quad \beta_{\mathrm{i}} \in \mathrm{K}, \quad \mathrm{x}_{\mathrm{k}} \in \mathrm{X} .
\end{gathered}
$$

Theorem 
Assume that $\sigma \in S_{n}$ be a cycle of order $n$ and $\theta$ be an automorphism of $\mathrm{F}$ induced by $\sigma$.

If $\bar{\theta}$ is an automorphism of $\mathrm{L}_{\mathrm{m}}$ induced by $\theta$ then every fixed point of $\bar{\theta}$ has the form

$$
\bar{\omega}+\bar{\theta}(\bar{\omega})+\bar{\theta}^{2}(\bar{\omega})+\ldots+\bar{\theta}^{\mathrm{n}-1}(\bar{\omega}),
$$

where $\bar{\omega}=\alpha \bar{x}_{k}+h, \quad \alpha \in K, x_{k} \in X, h \in L_{m}^{\prime}$.

Proof

Let $\bar{v} \in L_{m}$ be a fixed point of $\bar{\theta}$. We use induction on $m$. For $\mathrm{m}=1 \quad \mathrm{~L}_{1}=\mathrm{F} / \mathrm{F}^{\prime}$ is a free abelian Lie algebra. So by the Lemma the result is clear.

Suppose that the assertion is true for all positive integers less than $\mathrm{m}$. Let $\tilde{\theta}$ be an automorphism of $\mathrm{L}_{\mathrm{m}-1}$ induced by $\theta$.

By induction hypothesis the fixed points of the automorphism $\tilde{\theta}$ of the algebra $\quad \mathrm{L}_{\mathrm{m}-1}=\mathrm{F} / \delta^{\mathrm{m}-1} \mathrm{~F}$ are the elements of the form

$$
\widetilde{\omega}_{1}+\tilde{\theta}\left(\widetilde{\omega}_{1}\right)+\tilde{\theta}^{2}\left(\widetilde{\omega}_{1}\right)+\cdots+\tilde{\theta}^{\mathrm{n}-1}\left(\widetilde{\omega}_{1}\right)
$$

where $\widetilde{\omega}_{1}=\alpha \tilde{\mathrm{x}}_{\mathrm{k}}+\mathrm{h}_{1}, \quad \alpha \in \mathrm{K}, \mathrm{x}_{\mathrm{k}} \in \mathrm{X}, \mathrm{h}_{1} \in \mathrm{L}_{\mathrm{m}-1}^{\prime}$.

Let $\tilde{\mathrm{u}}$ be a fixed point of $\tilde{\theta}$ in $\mathrm{L}_{\mathrm{m}-1}$. Assume that $\tilde{\mathrm{u}}=$ $\widetilde{\psi}\left(\widetilde{\omega}_{1}\right)$, where

$\widetilde{\Psi}=\mathrm{I}+\tilde{\theta}+\tilde{\theta}^{2}+\cdots+\tilde{\theta}^{\mathrm{n}-1}$.

Since

$$
\mathrm{L}_{\mathrm{n}, \mathrm{m}-1}=\mathrm{F} / \delta^{\mathrm{m}-1} \mathrm{~F} \cong\left(\mathrm{F} / \delta^{\mathrm{m} F}\right) /\left(\delta^{\mathrm{m}-1} \mathrm{~F} / \delta^{\mathrm{m} F}\right),
$$

then the preimage of $\tilde{\mathrm{u}}$ in $\mathrm{F} / \delta^{\mathrm{m}} \mathrm{F}$ is of the form

$$
\mathrm{a}=\psi\left(\omega_{1}\right)+\mathrm{g}+\delta^{\mathrm{m}} \mathrm{F},
$$

where $g \in \delta^{\mathrm{m}-1} \mathrm{~F} / \delta^{\mathrm{m}} \mathrm{F}$. Then we have $\bar{\theta}(\overline{\mathrm{g}})=\overline{\mathrm{g}}$ in the algebra $\delta^{\mathrm{m}-1} \mathrm{~F} / \delta^{\mathrm{m}} \mathrm{F}$. By the Lemma the element $\overline{\mathrm{g}}$ has the form

$$
\overline{\mathrm{g}}=\bar{\omega}_{2}+\bar{\theta}\left(\bar{\omega}_{2}\right)+\bar{\theta}^{2}\left(\bar{\omega}_{2}\right)+\cdots+\bar{\theta}^{\mathrm{n}-1}\left(\bar{\omega}_{2}\right)
$$

where $\bar{\omega}_{2}=\beta \bar{b}, \quad \beta \in \mathrm{K}, \quad \overline{\mathrm{b}} \in \delta^{\mathrm{m}-1} \mathrm{~F} / \delta^{\mathrm{m}} \mathrm{F}$. Hence

$$
\mathrm{a}=\psi\left(\omega_{1}+\omega_{2}\right)+\delta^{\mathrm{m}} \mathrm{F}
$$

Now let $\bar{v}$ be a fixed point of $\bar{\theta}$ in $L_{m}$. The element $\bar{v}$ can be written as $\bar{v}=\bar{v}_{1}+\bar{v}_{2}$,

where $v_{1} \in F\left(\bmod \delta^{m-1} F\right), v_{2} \in \delta^{m-1} F$. Since $\bar{\theta}(\bar{v})=\bar{v}$ we get $\tilde{\theta}\left(\tilde{v}_{1}\right)=\tilde{v}_{1}$ and

$\bar{\theta}\left(\bar{v}_{2}\right)=\bar{v}_{2}$. By (2) and (3) we see that $\bar{v}_{1}+\bar{v}_{2}$ has the form

$$
\begin{gathered}
\overline{\mathrm{v}}_{1}+\overline{\mathrm{v}}_{2}=\bar{\psi}\left(\bar{\omega}_{1}+\bar{\omega}_{2}\right), \text { where } \bar{\omega}_{1}=\alpha \overline{\mathrm{x}}_{\mathrm{k}}+\mathrm{h}_{1}, \bar{\omega}_{2}= \\
\beta \overline{\mathrm{b}}, \quad \alpha, \beta \in \mathrm{K}, \mathrm{h}_{1} \in \mathrm{L}_{\mathrm{m}}^{\prime}, \overline{\mathrm{a}} \in \delta^{\mathrm{m}-1} \mathrm{~F} / \delta^{\mathrm{m}} \mathrm{F} .
\end{gathered}
$$

It can be easily seen that every element of the form

is a fixed point of $\bar{\theta}$.

\section{REFERENCES}

[1] R.M. Bryant, On the fixed points of finite group acting on a free Lie algebra. J. London Math Soc., 43(2): 215-224, 1991.

[2] R.M. Bryant, A.I. Papistas, On the fixed points of a finite group acting on a relatively free Lie algebra, Glasg Math J.,42:167-181, 2000.

[3] V. Drensky, Fixed algebras of residually nilpotent Lie algebras. Proc Amer Math Soc., 120: 1021-1028, 1994.

[4] N.Ekici, D.Sönmez, Fixed points of IA- endomorphisms of a free metabelian Lie algebra. Proc Indian Acad Sci (Math Sci. ),121(4):405416, 2011.

[5] T. Witold, Fixed points of automorphisms preserving the length of words in free solvable groups Arch. Math., 99:425-432, 2012. 